\title{
Thick-film force, slip and temperature sensors for a prosthetic hand
}

\author{
A Cranny, D P J Cotton, P H Chappell, S P Beeby and N M White
}

Electronic Systems Design Group, School of Electronics and Computer Science, University of Southampton, Southampton, Hampshire, SO17 1BJ, UK

E-mail: awc@ecs.soton.ac.uk

Received 30 September 2004, in final form 6 January 2005

Published 4 March 2005

Online at stacks.iop.org/MST/16/931

\begin{abstract}
Thick-film static and dynamic force sensors have been investigated for their suitability to measure the grip forces exerted upon an object held by a prosthetic hand, and to detect and correspondingly react to the possible slip of a gripped item. The static force sensors exploit the piezoresistive characteristics of commercially available thick-film pastes whilst the dynamic slip sensors utilize the piezoelectric behaviour of proprietary PZT (lead zirconate titanate) pastes. The sensors are located upon stainless steel cantilever type structures that will be placed at the fingertips of each digit of the prosthetic hand. Temperature sensors are also included to provide temperature compensation for the force sensors and to prevent accidental thermal damage to the prosthesis. Results have shown that the static force sensor is capable of measuring fingertip forces in excess of $100 \mathrm{~N}$, with an electrical half-bridge configuration sensitivity approaching $10 \mu \mathrm{V} \mathrm{V}^{-1} \mathrm{~N}^{-1}$ (with scope for improvement) and maximum hysteresis below $4 \%$ of full scale, depending on the manner in which the cantilever sensor array is attached to the finger. Failure in the bonding mechanism that secures the PZT layer to the stainless steel cantilever meant that the proposed dynamic force sensor could not be evaluated. However, investigations using the same sensor design fabricated on an alumina substrate have shown the potential of the PZT dynamic force sensor to measure the vibration and hence potentially operate as a slip sensor.
\end{abstract}

Keywords: artificial hand, force sensor, piezoelectric, piezoresistance, prosthesis, slip sensor, thick-film

(Some figures in this article are in colour only in the electronic version)

\section{Introduction}

A problem with the majority of prosthetic devices is the lack of any form of sensory feedback. In the case of a prosthetic hand this has the consequence that the user is unable to 'feel' an item held by the hand. In certain situations this could prove to be detrimental. For example, the lack of feedback on the force exerted during a grip posture could result in the crushing of a gripped item. Furthermore, the user would be unaware if he were holding a very hot or very cold item, possibly resulting in damage to the prosthesis or to the user. More generally, the inability to monitor the grip forces applied to a grasped object means that the user cannot be totally sure of the security of the grip and could be unaware (or have too little time to respond) should the object begin to slip from his grasp.

A possible solution to these problems is to include some form of multi-parameter sensing system within the prosthetic device. For the prosthetic hand, such a system might comprise a number of force sensors to monitor and adjust the grip strength and also include thermal sensors to measure the temperature of a grasped object. The system does not provide feedback to the operator but would respond automatically, taking preventative action should an object begin to slip from the grip of the prosthesis or was deemed to be of a temperature beyond the operating range of the prosthesis. 
Table 1. Requirements for a prosthetic hand force sensor.

\begin{tabular}{ll}
\hline Resolve forces up to $100 \mathrm{~N}$ & Not susceptible to EM interference \\
High sensitivity to small forces & Not easily damaged by large impact forces \\
Integral power supply & Robust \\
Lightweight & Service period of six months \\
Low cost & Simplicity in construction and mounting \\
Little hysteresis & Small size with an area less than $100 \mathrm{~mm}^{2}$ \\
Low power consumption & Thin in depth for mounting on fingers and palm \\
\hline
\end{tabular}

To monitor the grip strength and to detect the onset of slip requires a sensor system that comprises both static and dynamic force sensitive elements. A brief examination of the literature shows that a large, disparate range of sensor technologies and techniques have been evaluated for their suitability in such systems, spanning acoustic [1], capacitive [2, 3], Hall effect [4, 5], optical [6] and piezoresistive [7-9].

A suitable sensor fabrication technology for use in a prosthetic hand should demonstrate low temperature drift, good accuracy and good repeatability. In addition, the requirements listed in table 1 are also highly desirable, most noticeably the low power consumption and being lightweight. Many sensor enabling technologies meet some of the requirements listed but few, if any, meet them all.

Previous work has identified thick-film technology as a suitable fabrication technique for the production of both static and dynamic force sensors. For those not familiar with the technology, a good overview is given by Holmes and Loasby [10]. For example, low cost force sensors can be achieved by exploiting the piezoresistive characteristics of commercially available thick-film resistive pastes [11-15]. These materials, when printed and fired with electrically conductive end terminations in a planar configuration, form electrical resistors with typical post-processed thicknesses of the order of 10 to $12 \mu \mathrm{m}$ that exhibit proportional changes in their resistance with applied strain. Thick-film piezoelectric materials such as PZT (lead zirconate titanate) can be used to construct vibration sensors [16-18], which may be used for the slip detection. The simplicity of thick-film technology also means that production times are relatively short, enabling the quick prototyping and evaluation of candidate sensor structures.

\section{Description of the prosthetic hand}

The prosthetic hand used in this project is a prototype myoelectrically driven device designed at the University of Southampton and described elsewhere [19]. The skeletal structure of the hand is shown in figure 1 . In use the hand is contained within a synthetic skin to give a more anthropomorphic look. This also has the additional benefit of providing environmental protection to the sensors located on the skeletal structure. The hand is controlled by the electrical signals produced by any convenient flexor-extensor muscle pair. Signals from these muscles form the inputs to an intelligent, state driven controller which interprets this information before moving the digits of the hand into one of several prehensile positions including hold, squeeze, grip and release, as well as some of the more common hand postures [20-22]. To perform these functions, each finger is individually controlled by its own dedicated motor, allowing

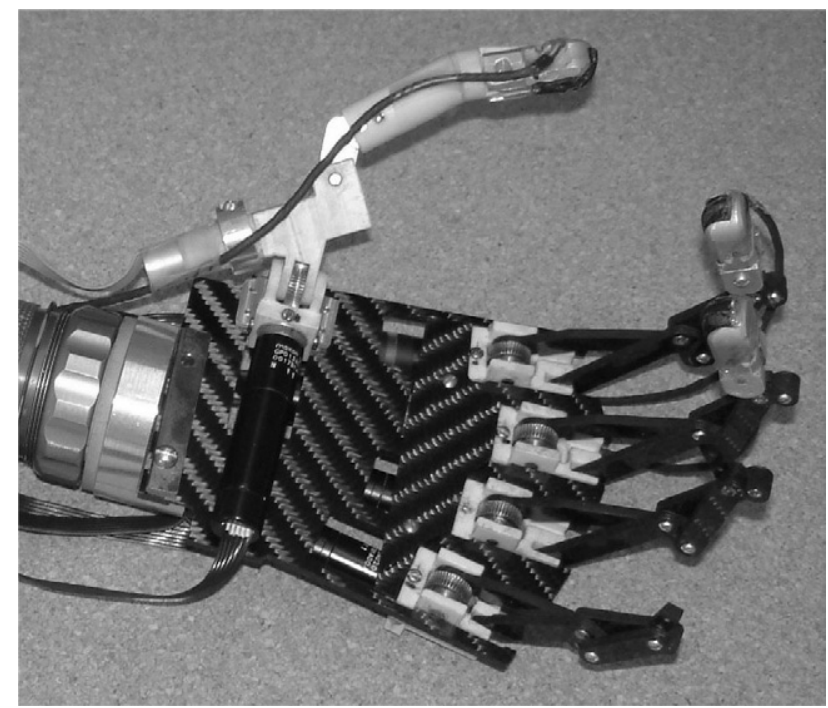

Figure 1. Skeletal structure of the prototype prosthetic hand showing some early photo/acoustic sensors for grip detection located on the fingertips and thumb.

independent flexion (closing) and extension (opening) of each mechanical digit.

The fingers are constructed from a number of interconnecting links, which when driven by the motors cause the fingers to close or open in a natural anthropomorphic pattern [23]. The finger links and palm of the hand are fabricated from a carbon fibre epoxy composite to reduce the overall mass of the hand. The thumb is manufactured from Hilube Vesconite ${ }^{\mathrm{TM}}$ for its greater mechanical strength and very low water absorption properties. The thumb is controlled by two orthogonal motors giving two degrees of freedom in movement, simulating the abduction, adduction, flexion and extension movements of the natural thumb. In conjunction with the four independently controllable fingers, the hand therefore has a total of six degrees of freedom allowing a range of natural grip postures to be adopted.

This paper describes the development of an array of sensors that includes a single static force sensor, a single dynamic force sensor and a single temperature sensor. These sensor devices are located on a cantilever type structure that mimics the shape of a human fingertip. A cantilever structure was chosen since this readily allows the conversion of fingertip forces to mechanical strain, which is easier to detect and measure than force itself. Each fingertip cantilever will be mounted at the distal end of each finger, symmetrically about the central long axis of the finger. This arrangement is intended to ensure a symmetrical strain distribution along the length of the cantilever when it is deflected and to help reduce force 
Force sensors for a prosthetic hand

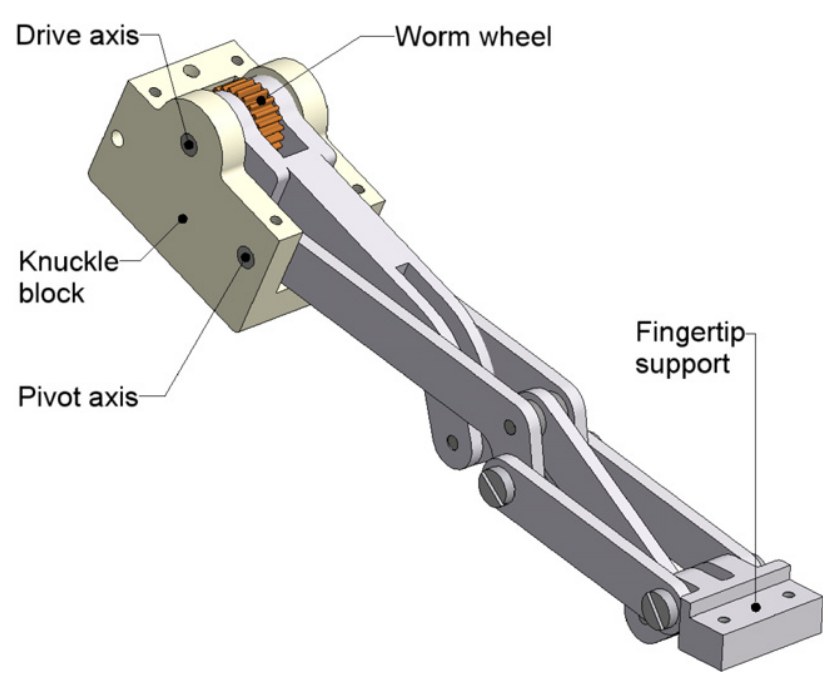

Figure 2. Modified finger link system. The knuckle block containing the motor and drive wheel assembly is shown on the left of the diagram, and the fingertip support is shown to the right.

measurement errors that may arise, for example, through twisting of the beam caused by an off-axis loading of the fingertip.

Unfortunately, the arrangement of the finger link system of the prototype prosthetic hand did not enable symmetrical mounting of the cantilever about the long axis. Hence, it was necessary to redesign the finger link system, as shown in figure 2. This new design comprises seven linked elements with the distal end being capable of supporting a number of different shaped cantilever structures. This finger has been modelled to ensure that the trajectory of the fingertip during opening or closing of the hand is not significantly different from that of the original version.

\section{Fingertip sensor array}

Each of the three different types of sensor included upon the fingertip cantilever is produced using thick-film technology. This production process involves the sequential printing of patterned layers of specialist pastes, the formulation of which define the electrical behaviour of that layer [10]. Conductive pastes, for example, contain a large proportion of metallic particles, whereas resistive pastes contain a blend of metal oxides and insulating materials, the ratio of which defines the resistivity of the paste. In general, thick-film pastes include a glass component as a binder and adhesion mechanism and as such are usually processed at high temperatures of the order of $850{ }^{\circ} \mathrm{C}$ to $950{ }^{\circ} \mathrm{C}$. Consequently the substrate material upon which the thick-film sensors are produced must be capable of withstanding these high processing temperatures. Conventionally ceramic materials such as alumina are used. However, for this application the production of sensors on an alumina cantilever would be inappropriate since this material is too brittle to withstand the bending forces applied to the fingertips when in use. Fortunately, particular grades of stainless steel have proved to be compatible with the thickfilm process $[13,18,24]$ and have therefore been considered here. Stainless steel also has the additional advantage that it may be machined and shaped more readily than alumina.

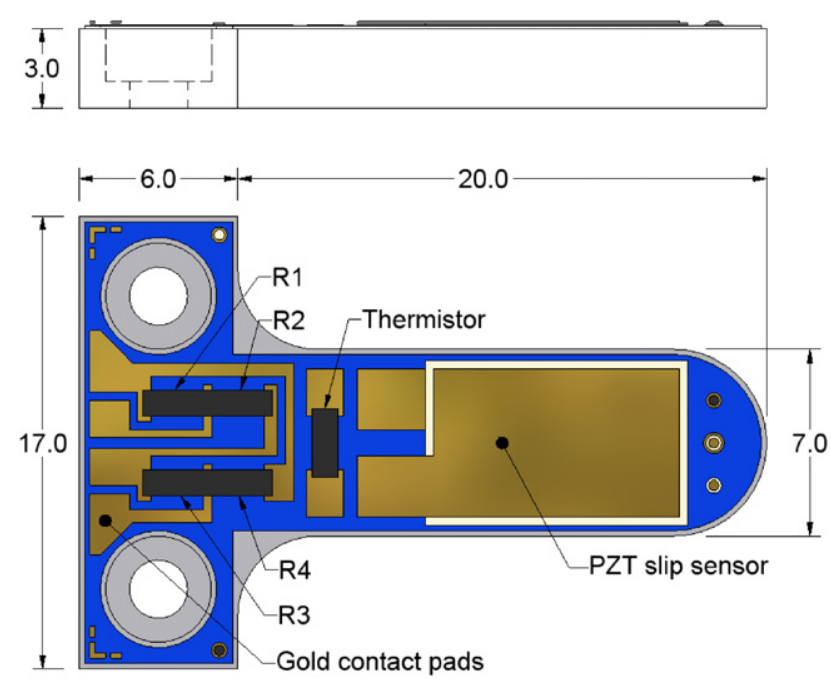

Figure 3. Dimensions of the fingertip cantilever ( $\mathrm{mm})$ and the location of sensors.

The dimensions of the fingertip cantilever and the locations of the various sensor structures are shown in figure 3. The cantilever is machined from grade 304 stainless steel and has a nominal thickness of $3 \mathrm{~mm}$, which may be changed (along with the beam width) to adjust the sensitivity and/or the measurement range of the device. The design differs from that of a traditional cantilever structure due to machining limitations that introduce a fillet where the beam joins the root of the structure. Ideally, the beam width should remain constant along its whole length.

Before printing the various thick-film pastes that form the force and temperature sensors, the surface of the stainless steel is first insulated using a compatible dielectric insulating paste (ESL 4916). This was deposited as two sequentially processed layers to an average thickness of $70 \mu \mathrm{m}$, where a single processing cycle involves the printing, drying and then high temperature firing of the patterned layer.

\subsection{Static force sensor}

The static force sensor exploits the piezoresistive effect exhibited by thick-film resistors [11-14]. In this effect, the resistance of a thick-film resistor changes in a linear manner with the strain experienced by the resistor. The magnitude of the strain sensitivity is given by the gauge factor, $G$, which is defined as the unit change in resistance per unit of strain applied:

$$
G=\frac{\delta R}{R} \frac{1}{\varepsilon} .
$$

Here, $\delta R$ is the change in resistance due to a strain, $\varepsilon$, applied to a resistor of unstrained resistance, $R$. Values for $G$ for thickfilm resistors depend upon the nominal sheet resistivity of the resistor paste, with typical values in the range 8 to 12 being reported [11-13]. This is much greater than that associated with conventional metal foil gauges $(G=2)$ and is due to the fact that the resistivity of a thick-film resistor is itself dependent upon strain $[25,26]$. Hence a thick-film resistor is a more sensitive strain sensing element than a conventional metal foil gauge. 


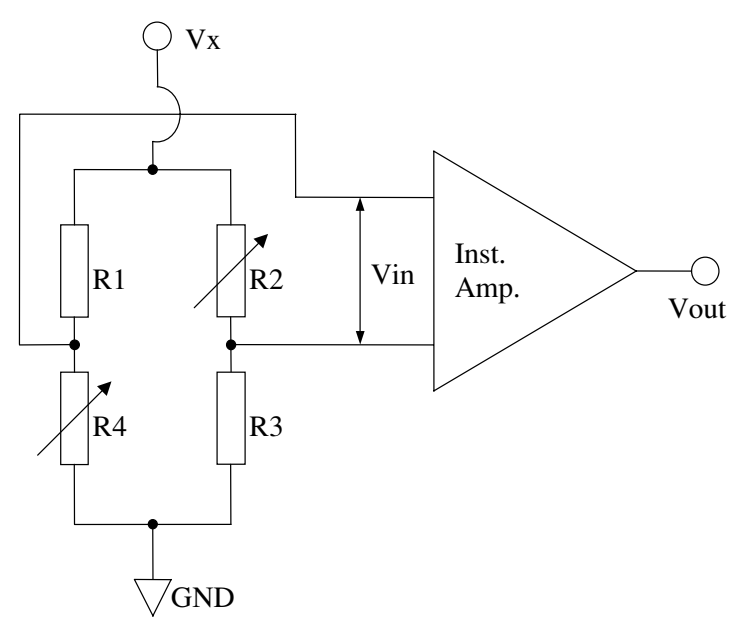

Figure 4. Half-bridge arrangement of thick-film resistors to produce a static force measurement circuit.

The static force sensor is located at the root of the beam (as shown in figure 3 ) and consists of four individual thickfilm resistors ( $R 1$ through $R 4)$ arranged on the cantilever in a classic resistance half-bridge circuit. Conventionally strain sensors are usually arranged in a Wheatstone bridge circuit with four active components located at the root of the cantilever and on both sides, thereby exploiting strains of compression and tension to maximize measurement sensitivity. However in this application only the top surface of the cantilever is used (to reduce costs and processing time) and therefore only tensioning strains are measured as the fingertip is deflected by a force.

The half-bridge circuit (depicted in figure 4 along with a suitable measurement circuit) was chosen since it readily gives a measure of the unit change in resistance. Two of the four resistors are located at the theoretical position of the maximum strain for a given beam deflection ( $R 2$ and $R 4)$ and serve as the active strain sensing components of the sensor. The remaining two resistors $(R 1$ and $R 3)$ act as the passive components of the bridge circuit and as such should remain insensitive to the beam deflection. Consequently, these two resistors are located on the body of the structure away from the beam itself, where it is firmly attached to the fingertip support. In theory, because this part of the structure should remain rigid when the beam is deflected by a force, these two resistors should experience zero strain (or at the very least, a constant strain level). The passive resistors are also positioned close to the active resistors $(R 2$ and $R 4$ ) so that they all experience the same local temperatures, ensuring that first-order temperature compensation is achieved more readily.

Other locations for the placement of the passive components were considered, such as the distal end of the fingertip cantilever, but were subsequently rejected since although these locations exhibit a minimal strain when a force is applied to the fingertip, the level of strain experienced would however not remain constant with changing forces. In addition, and from a fabrication perspective, printing all four thick-film resistors as close as possible to each other improves the repeatability in electrical characteristics between the individual resistors such as the temperature coefficient of resistance (TCR) and the gauge factor $(G)$. Matching the resistor characteristics in this manner serves to improve the overall sensitivity of the bridge circuit.

The resistor paste (ESL 3914) was printed and fired over a pattern of gold conductive tracks (ESL 8836) which define the dimensions of the individual resistors $(2 \mathrm{~mm} \times 1 \mathrm{~mm})$. The conductor track pattern also forms the electrical interconnect for the half-bridge circuit and provides a solderable interface for external connection. The resistor paste chosen has a nominal sheet resistivity of $10 \mathrm{k} \Omega / \square$, resulting in post processed resistance values of approximately $20 \mathrm{k} \Omega$.

When a force is exerted upon the fingertip, the cantilever is deflected. Standard beam theory predicts that the force dependent strain $\varepsilon(F)$ at the root of a true cantilever beam (the position where it is secured and the region where $R 2$ and $R 4$ are located) is given by [27]

$$
\varepsilon(F)=\frac{6 F L}{E b h^{2}} .
$$

Here, $F$ is the force at the tip of the beam, $E$ is the material parameter Young's modulus of the stainless steel beam, and $L$, $b$ and $h$ are respectively the beam length, width and thickness. For the beam dimensions shown in figure 3, and for a value for $E$ of $1.9 \times 10^{11} \mathrm{~N} \mathrm{~m}^{-2}$, a force of $100 \mathrm{~N}$ at the beam tip should produce a theoretical maximum strain at the beam root of approximately 1 milli-strain, which is within the elastic limit of the grade of stainless steel used and within the workable range of the thick-film materials employed to produce the various sensors [28, 29].

The output of the resistance half-bridge circuit, normalized with respect to the bridge excitation voltage $V_{x}$, is given by

$$
\frac{V_{\mathrm{out}}}{V_{x}}=\frac{\delta R}{2 R} .
$$

This expression assumes that all the four resistors have identical values for their respective unstrained resistances of $R$ and that they all exhibit the same level of strain sensitivity (i.e. have the same gauge factor, $G$ ). Combining equations (1), (2) and (3) yields an expression directly relating the bridge output signal to the force applied at the beam tip, from which a theoretical value for the force measurement sensitivity can be derived:

$$
\frac{V_{\text {out }}}{V_{x}} \frac{1}{F}=\frac{3 G L}{E b h^{2}} .
$$

Estimating the value of the gauge factor of the thick-film resistors to be 10, equation (4) yields a value for the force measurement sensitivity of approximately $50 \mu \mathrm{V} \mathrm{V}^{-1} \mathrm{~N}^{-1}$. For a $3 \mathrm{~V}$ bridge supply, the theoretical measurement sensitivity would therefore be of the order $150 \mu \mathrm{V} \mathrm{N}^{-1}$, which is readily detectable above the background noise with a good quality instrumentation amplifier.

\subsection{Dynamic force sensor}

The dynamic force sensors are essentially vibration sensors and are fabricated from PZT that has been rendered into a form that is suitable for thick-film printing in our laboratories $[30,31]$. This material is piezoelectric, i.e. it generates an electric charge on its surface when mechanically deformed. Multiple layers of this material are printed and fired upon an underlying gold conductive electrode pad (ESL 8836) to a 
Force sensors for a prosthetic hand

thickness of approximately $100 \mu \mathrm{m}$ before a final conductive electrode is printed upon the top surface, forming a capacitor structure. The devices are then individually poled in a $\mathrm{dc}$ electric field at elevated temperatures to initiate their piezoelectric behaviour. An indication of the strength of the piezoelectric behaviour achieved can be determined by the measurement of the $d_{33}$ piezoelectric coefficient - a measure of the charge produced per unit applied stress in the same axis [32]. After polarization, these thick-film devices have been shown to exhibit $d_{33}$ coefficients up to $110 \mathrm{pC} \mathrm{N}^{-1}$ depending on the poling conditions, the substrate material, the composition of the electrode material and the formulation of the PZT paste [30, 31, 33]. Each dynamic force sensor covers a large proportion of the available area of the fingertip cantilever and is used to detect any rapid variations in the force over the fingertip surface, or vibration, which could be indicative of an object slipping from grasp. The change in force over the surface area of a single piezoelectric sensor causes a proportional change in the charge distribution of the PZT layer which is readily measured by an impedance balanced charge amplifier.

\subsection{Temperature sensor}

The temperature of an object held within the prosthetic hand is determined by monitoring the change in the resistance of a thick-film thermistor paste (ESL PTC2611) printed in the space between the two force sensors. This particular paste has a low value for sheet resistivity (nominally $10 \Omega / \square$ ) but demonstrates a highly linear relationship between resistance and temperature. It is specified as having a positive temperature coefficient of resistance of the order of $4100 \pm$ $500 \mathrm{ppm}{ }^{\circ} \mathrm{C}^{-1}$ over the temperature range $-55^{\circ} \mathrm{C}$ to $+125^{\circ} \mathrm{C}$. The printed thermistor has dimensions of $2 \mathrm{~mm} \times 1 \mathrm{~mm}$ and should therefore have a post processed resistance value of approximately $20 \Omega$ at room temperature. In addition to monitoring the temperature of held objects, this sensor also provides temperature compensation for the various force sensors.

\section{Experimental results}

\subsection{Static force sensor}

The static force sensor was evaluated by securing the cantilever structure to a purpose built test rig and loading the fingertip with masses up to a total of $1 \mathrm{~kg}$ in $100 \mathrm{~g}$ increments whilst measuring the change in bridge output voltage. The average change in the output computed from a number of samples is shown graphically in figure 5 . The data have been normalized with respect to the bridge excitation voltage and offset values have been removed. The error bars in the figure represent the maximum and minimum deviations in the data set. Their observed increase in span with increasing force can be partly attributed to the difficulty in repeatedly placing a greater number of individual masses at the same point on the fingertip. The figure shows that the force sensor exhibits a reasonably linear relationship over the range investigated, with an average sensitivity of $6.4 \pm 0.3 \mu \mathrm{V} \mathrm{V}^{-1} \mathrm{~N}^{-1}$ (a statistical variation of $5 \%$ ). This is nearly a factor of eight times lower than the force sensitivity predicted using equation (4).

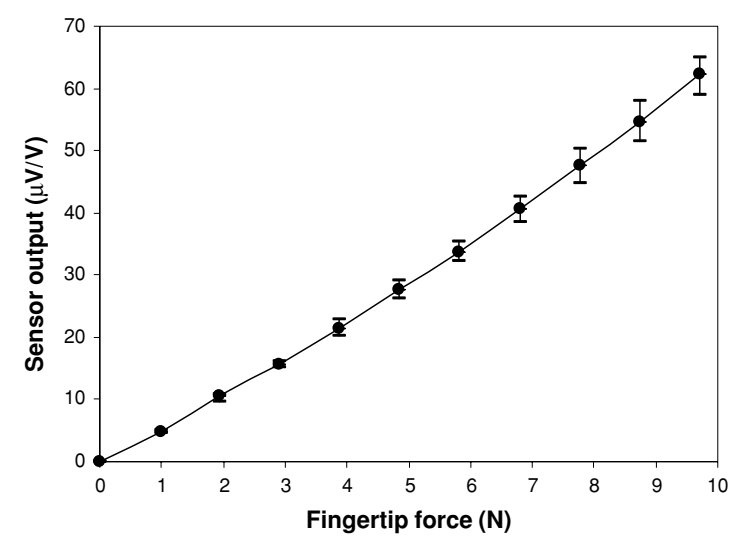

Figure 5. Static force sensor average normalized output over low force range.

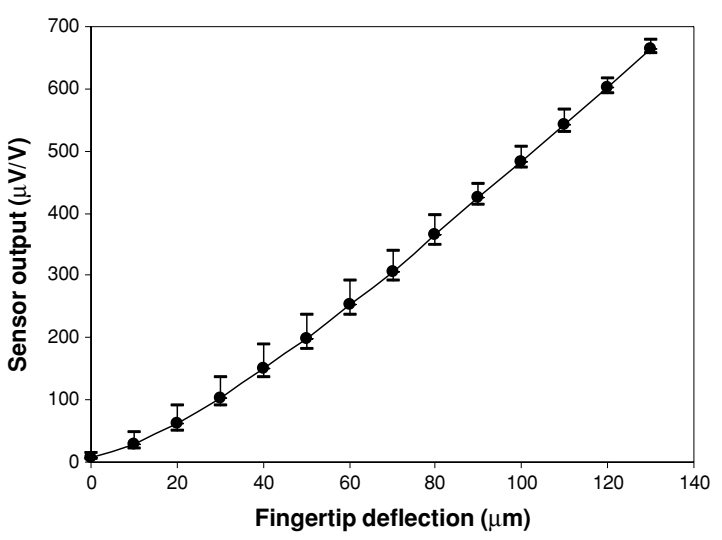

Figure 6. Static force sensor average normalized output as a function of fingertip deflection.

To investigate the sensor response over the full force range, a graduated micrometer was used to deflect the beam tip. This technique was employed due to the impracticality of balancing a large number of masses at the tip of the cantilever structure. The average response from a number of devices is shown in figure 6. Again, the data have been normalized with respect to the bridge excitation voltage and offset values have been removed. Tip deflections up to a maximum of $130 \mu \mathrm{m}$ are shown in the figure, which is equivalent to a theoretical beam tip force of approximately $146 \mathrm{~N}$ (for an ideal cantilever beam [27]). The figure shows that in general the sensor output changes in a linear manner with beam tip deflection, with an equivalent sensitivity of $6.5 \pm$ $0.2 \mu \mathrm{V} \mathrm{V}^{-1} \mathrm{~N}^{-1}$ calculated over the more linear portion of the curve (a statistical variation of $3 \%$ ). This value is in good agreement with the corresponding value calculated for the investigation over the lower force range.

\subsection{Effect of load position}

A potential problem with the static force sensor design in its present form is its inability to distinguish between two different forces contacting the fingertip structure at two different positions. For example, the sensor is unable to distinguish between a $50 \mathrm{~N}$ force located at the beam tip and a $100 \mathrm{~N}$ force acting half way along the beam, since both produce the same strain at the sensor location as a consequence of the moments 
Table 2. Force measurement sensitivity $\left(\mu \mathrm{V} \mathrm{V}-1 \mathrm{~N}^{-1}\right)$ and bridge offset voltage $\left(\mu \mathrm{V} \mathrm{V}^{-1}\right)$ as a function of the distance (mm) that force is applied from the tip of the cantilever beam.

\begin{tabular}{lcccccccc}
\hline Distance & 1 & 2 & 3 & 4 & 5 & 6 & 7 & 8 \\
Sensitivity & 7.0 & 6.6 & 6.4 & 6.0 & 5.2 & 4.9 & 4.3 & 3.8 \\
Offset & -1.30 & -0.04 & -0.72 & -0.49 & -0.22 & 0.55 & -0.59 & 0.23 \\
\hline
\end{tabular}

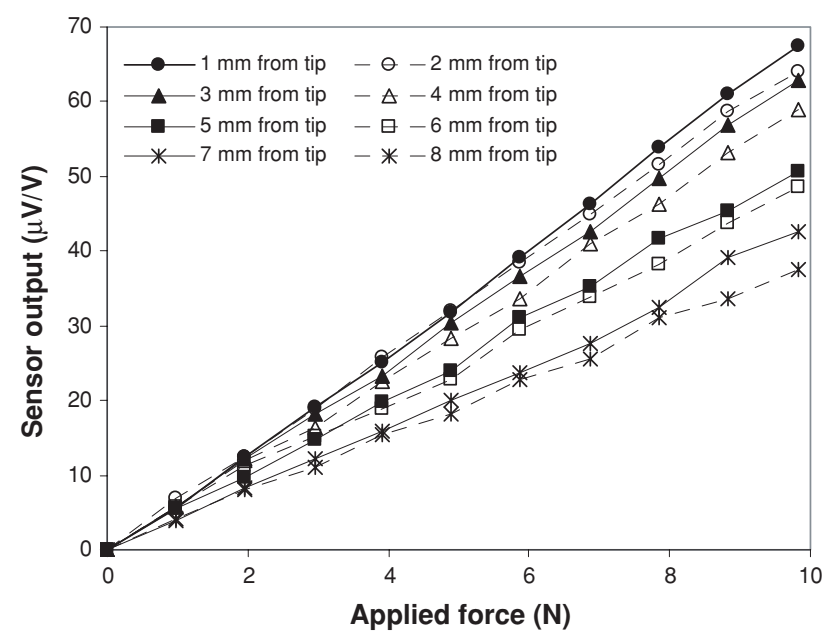

Figure 7. Static force sensor normalized output as a function of loading position.

being identical. This is illustrated in the response curves of figure 7 which show the sensor output, normalized with respect to the bridge excitation voltage, as a function of the applied force at various distances from the beam tip.

The force measurement sensitivity can be determined directly from the slopes of the curves shown in the figure. These values are listed in table 2 along with values for the bridge offset voltage normalized with respect to the bridge excitation voltage. The table of values shows that the measurement sensitivity decreases in a linear manner as the force is moved further away from the tip. This is expected since the surface strain at the beam root varies in a linear manner with the distance that the deflecting force acts from the beam root. With this in mind we would expect the intersections of the individual curves with the ordinate axis at $10 \mathrm{~N}$ to be equally spaced. The fact that they are not quite so can be attributed to a number of factors including uncompensated temperature induced effects (such as bridge supply fluctuations) and simple measurement uncertainty in the exact position of the force along the beam length.

Performing simple linear regression analysis on the figures in table 2 reveals that the sensitivity of the force sensor decreases at a rate of approximately $-0.46 \mu \mathrm{V} \mathrm{V}^{-1} \mathrm{~N}^{-1} \mathrm{~mm}^{-1}$ (a $6 \%$ decrease in sensitivity per millimetre distance from the beam tip end). In practice, this positional variation in the measurement sensitivity may not be a problem since the envisaged use of the static force sensor is to monitor and adjust grip to prevent object slip rather than to measure absolute forces involved in grip postures. However, it is still possible to use this device to measure absolute force values by the addition of an extra mechanical structure, so arranged that it always couples contacting forces with the tip of the cantilever beam.

Table 2 also shows that there is some variation in the bridge circuit offset voltage with the distance from the beam

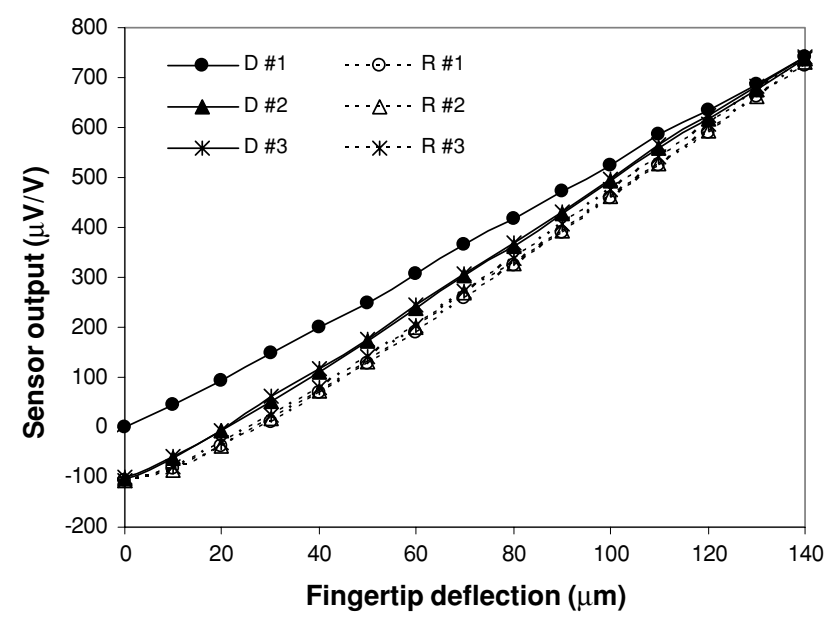

Figure 8. Normalized sensor response over a succession of fingertip deflection (D) and relaxation (R) trials.

tip that the force is applied, though this does not exhibit a quantifiable trend. This is not considered to be an issue since any change in the offset voltage may be compensated through suitable electronics in the measurement circuit. For example, the offset voltage may be measured and automatically zeroed each time a finger is in the fully open position and the force sensor is not subject to any external load.

\subsection{Hysteresis effects}

Hysteresis effects in the response of the static force sensor have been investigated by recording the sensor bridge output voltage as the tip of the cantilever beam is first deflected by a graduated micrometer and then as it is relaxed back as the micrometer is unwound. Figure 8 shows a typical set of responses over three successive trials of beam deflection and relaxation. The results shown have been obtained by taking the difference in the sensor response between the un-deflected reading of the first deflection trial (i.e. the initial unstrained value) and all subsequent measurements, and then normalising these values with respect to the bridge excitation voltage. Values for the force measurement sensitivity, bridge offset voltage and maximum hysteresis levels have been calculated from the slopes and intercepts of the curves and are listed in table 3. Here, and throughout this section, the maximum hysteresis level is calculated as the largest difference recorded between the sensor responses for the same micrometer setting, expressed as a percentage of the average response range recorded during both deflection and relaxation of the beam in the same experiment trial. In the case of the measurement sensitivity figures, these have been converted from a deflection based value to a force equivalent figure using the theoretically derived association that a $140 \mu \mathrm{m}$ deflection at the tip of an ideal cantilever of similar dimensions is equivalent to a beam tip force of $157 \mathrm{~N}$ [27]. 
Table 3. Force measurement sensitivity $\left(\mu \mathrm{V} \mathrm{V}^{-1} \mathrm{~N}^{-1}\right)$, bridge offset voltage $\left(\mu \mathrm{V} \mathrm{V}^{-1}\right)$ and maximum response hysteresis over three successive trials of beam deflection $(\mathrm{D})$ followed by beam relaxation $(\mathrm{R})$.

\begin{tabular}{lcccccc}
\hline & $\mathrm{D} \# 1$ & $\mathrm{R} \# 1$ & $\mathrm{D} \# 2$ & $\mathrm{R} \# 2$ & $\mathrm{D} \# 3$ & $\mathrm{R} \# 3$ \\
\hline Sensitivity & 6.1 & 7.1 & 7.0 & 7.1 & 7.0 & 7.2 \\
Offset & -11.31 & -159.67 & -126.55 & -157.38 & -121.21 & -153.05 \\
Max. hysteresis & \multicolumn{2}{c}{$17.4 \%$} & \multicolumn{2}{c}{$4.9 \%$} & \multicolumn{4}{c}{$4.7 \%$} \\
\hline
\end{tabular}

Table 4. Force measurement sensitivity $\left(\mu \mathrm{V} \mathrm{V}^{-1} \mathrm{~N}^{-1}\right)$, bridge offset voltage $\left(\mu \mathrm{V} \mathrm{V}^{-1}\right)$ and maximum response hysteresis over four successive trials of cantilever deflection (D) followed by beam relaxation $(\mathrm{R})$ when the cantilever was glued to its support block.

\begin{tabular}{lcccccccc}
\hline & $\mathrm{D} \# 1$ & $\mathrm{R} \# 1$ & $\mathrm{D} \# 2$ & $\mathrm{R} \# 2$ & $\mathrm{D} \# 3$ & $\mathrm{R} \# 3$ & $\mathrm{D} \# 4$ & $\mathrm{R} \# 4$ \\
\hline Sensitivity & 9.5 & 10.0 & 9.7 & 9.9 & 9.7 & 10.0 & 9.7 & 9.8 \\
Offset & -17.78 & -55.11 & -38.44 & -55.12 & -36.69 & -53.60 & -41.76 & -51.93 \\
Max. hysteresis & \multicolumn{2}{c}{$5.8 \%$} & \multicolumn{2}{c}{$3.3 \%$} & \multicolumn{2}{c}{$3.6 \%$} & \multicolumn{2}{c}{$2.6 \%$} \\
\hline
\end{tabular}

Figure 8 shows that there is a significant change in the sensor response between the very first set of deflection data and any subsequent measurement. However, this large difference in the sensor response is not indicative of true hysteresis since all subsequent measurements of the sensor response after the first deflection trial show a common change in the unloaded offset value which exaggerates any differences (cf table 3 ). A true indicator of hysteresis in the system can be ascertained by examining the difference in the sensor response between the second and third trials. Over the deflection range investigated this equates to a maximum hysteresis value equivalent to approximately $5 \%$ of the full scale response.

Table 3 shows that the recorded measurement sensitivity is somewhat higher than that previously observed and that there is a significant difference in the sensitivity between the first measurement trial (D\#1) and the remaining trials. The reasons for these large discrepancies in the sensor response between the first deflection trial and all subsequent trials may be attributed to the method by which the cantilever device is attached to the test rig. It has been hypothesized that the observed effect is due to loosening or re-seating of the two bolts that secure the device caused by mechanical effects during the first deflection trial. If the force securing these bolts changes then the local surface strain in the region between the two bolts will also show a change. Since this is the region where the passive thick-film resistors of the bridge circuit are located ( $R 1$ and $R 3)$, any change in strain in this location will result in a change in their resistance and hence a change in the bridge signal offset value with a corresponding change in the measurement sensitivity and calculated hysteresis.

The evidence from a second investigation would seem to support this hypothesis. Here the cantilever beam has been attached to the test rig by a cyanoacrylate adhesive rather than being bolted in place. The results (shown in figure 9 and table 4) show that in this instance there is no dramatic change in the unloaded offset value between the measurement trials and the force measurement sensitivity figures for all the curves are generally in good agreement, with an average value of $9.8 \pm 0.2 \mu \mathrm{V} \mathrm{V}^{-1} \mathrm{~N}^{-1}$. This is as much as $50 \%$ greater than had been previously observed when the cantilever was bolted to a support structure. The computed levels of hysteresis between responses under these conditions are also lower, due to improved repeatability in the measurement sensitivity and bridge offset values, with typical maximum values of the order of $3 \%$ to $6 \%$ of the full scale response.

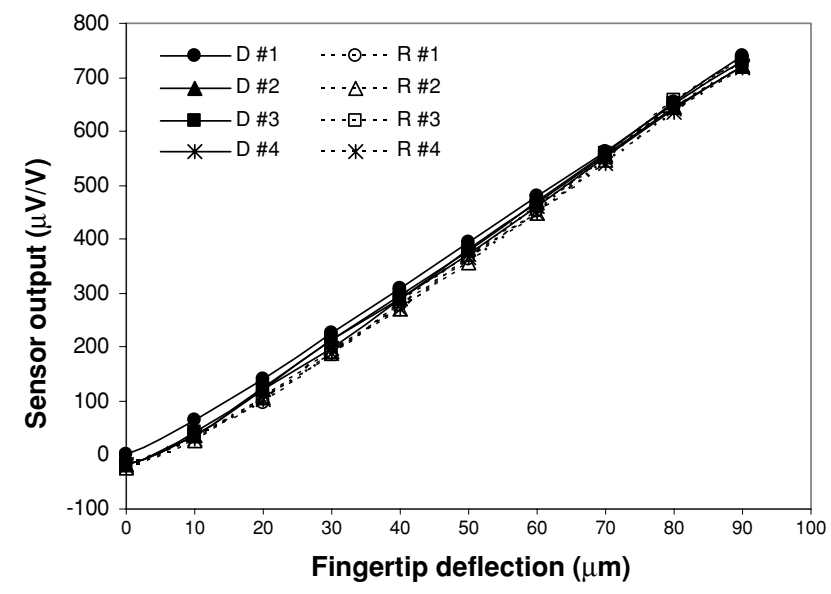

Figure 9. Normalized sensor response over successive trials of deflection (D) and relaxation (R) when the fingertip cantilever is glued to the test rig.

\subsection{Dynamic force sensor}

During the fabrication of the fingertip thick-film sensors, problems were encountered with the adhesion of the PZT layer. The material printed correctly, forming well-defined patterned layers that retained their shape when dried. However, during and after the firing process (peak temperature of $880{ }^{\circ} \mathrm{C}$ for $10 \mathrm{~min}$ in a belt furnace) the PZT layers fractured and delaminated from their underlying electrode patterns. In some instances the results were dramatic: the PZT layer removed both the supporting conductor layer and the underlying dielectric insulation layer with it, exposing the surface of the stainless steel substrate. As a consequence the dynamic force sensor could not be implemented directly on the fingertip cantilever.

The reasons for the PZT layer failing to bond to the gold conductor electrode are not at present fully understood. A previous work has shown that this exact formulation of PZT paste shows no adhesion problems with thick-film conductor materials printed on $96 \%$ alumina [30, 31, 33] and other grades of insulated stainless steel [34, 35]. A possible explanation is a build up of internal stresses due to a combination of mismatches in the thermal expansion coefficients of the stainless steel substrate and the individual thick-film layers, though samples of PZT paste printed directly 


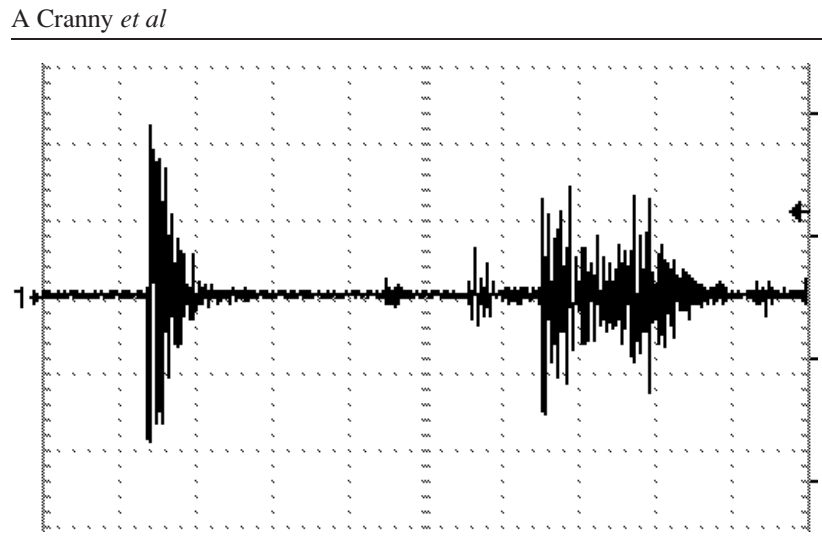

Figure 10. Oscilloscope trace of output from charge amplifier as $100 \mathrm{~g}$ weight is dropped onto the surface of a PZT slip sensor and then allowed to roll over its surface. Horizontal axis: $50 \mathrm{~ms}$ per division; vertical axis: $2 \mathrm{~V}$ per division.

onto the stainless steel showed no adhesion problems and the dielectric insulating paste chosen was thermally matched to the grade of stainless steel used. A second reason could involve chemical attack of the interfacial bond between the PZT layer and the gold conductor by reactive components out-gassed from the stainless steel during the firing process.

To demonstrate the potential of thick-film PZT dynamic force sensors to act as slip detectors, exact copies of the sensor design were fabricated on $96 \%$ alumina tiles $(0.625 \mathrm{~mm}$ thick), patterned with laser scribe lines to allow individual devices to be 'snapped out' and glued to the fingertip cantilever surface. These devices exhibited no problems during their manufacture. Once fabricated, individual sensor elements were poled in a dc electric field (field strength of approximately $4 \mathrm{MV} \mathrm{m}^{-1}$ ) at a temperature of $150{ }^{\circ} \mathrm{C}$ for $30 \mathrm{~min}$ and then allowed to cool to room temperature whilst the electric field was maintained across the PZT layer. The $d_{33}$ piezoelectric coefficient of a number of samples was then measured using a Take Control PM35 piezometer, yielding values in the range 50 to $55 \mathrm{pC} \mathrm{N}^{-1}$.

Figure 10 shows a typical response from one of these devices when connected to a simple charge amplifier. Here, a small $100 \mathrm{~g}$ weight has been dropped onto the top surface of a cantilever lying flat on an inclined surface. The initial moment of impact can be seen on the left of the trace as well as a vibration signal on the right side of the trace as the weight slips over the surface of the dynamic sensor. Both signals are readily detectable above the background noise level and demonstrate the potential of the sensor to detect the moment of first contact the hand makes as it closes on an object as well as its ability to detect the onset of slip. Indeed, it may be possible to further analyse the signal to determine physical characteristics of the gripped object. For example, it may be possible to determine whether an object is solid or hollow, or to determine its coefficient of friction. This information could then be used in deciding what level of force the fingers need to apply to maintain a secure grip.

\subsection{Temperature sensor}

The temperature sensor has two distinct purposes. Firstly it is used to measure the temperature of a gripped object to prevent accidental damage to the prosthesis. Secondly,

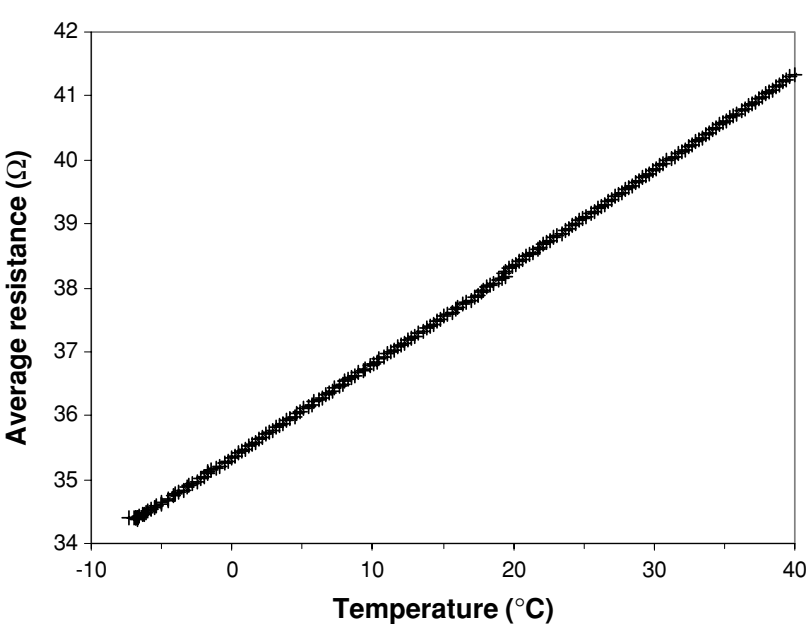

Figure 11. Thermistor resistance as a function of temperature.

it will be used to provide temperature compensation to the measurements obtained from the two force sensors (primarily the static force sensor) should this be found to be necessary. Considering the static force sensor, if it is assumed that the temperature coefficients of resistance of the four individual resistors are closely matched then, in theory, the output of the bridge-circuit should be temperature independent since strain induced ratiometric changes in resistances are maintained with temperature. However, it has been demonstrated that the gauge factor of thick-film resistors exhibits a temperature dependency [29] and therefore we would expect the force measurement sensitivity to exhibit some variation with temperature.

The room temperature resistance of the post processed thermistors was nearly twice as large as expected, with an average value of $39.0 \pm 5.0 \Omega$ at $25^{\circ} \mathrm{C}$. This was attributed to a thinner printed film than recommended for this material, but was not considered a problem. In fact a higher nominal resistance is advantageous since it permits an easier and potentially more accurate measurement of resistance changes.

To characterize the temperature dependence of the thickfilm thermistors, the resistances of a number of samples were measured individually in a Vötsch VT4021 environmental chamber between $-10{ }^{\circ} \mathrm{C}$ and $+40{ }^{\circ} \mathrm{C}$. The local temperature experienced by each thermistor was determined by measuring the resistance of a calibrated commercial thin-film Pt100 temperature sensor glued to the thermistor surface. The average measured resistance as a function of the local temperature is reproduced in figure 11, which shows a highly linear relationship of the form

$$
R_{(T)}=R_{(0)}(1+\alpha T) .
$$

Here, $R_{(T)}$ is the resistance at a temperature $T, R_{(0)}$ is the resistance at $0{ }^{\circ} \mathrm{C}$ and $\alpha$ is the temperature coefficient of resistance. From the results, average values for $\alpha$ and $R_{(0)}$ were computed: $\alpha=4230 \pm 50 \mathrm{ppm}^{\circ} \mathrm{C}^{-1}$ and $R_{(0)}=35.4 \pm$ $5.5 \Omega$. The large spread in the value for $R_{(0)}$ reflects the large variation in post-processed resistance values for these devices; an artefact of the thick-film printing process. This is not considered a problem since individual thermistor devices may be balanced in a suitable bridge circuit in operation. The small variation in the value for $\alpha$ (approximately 1.2\%) means that 

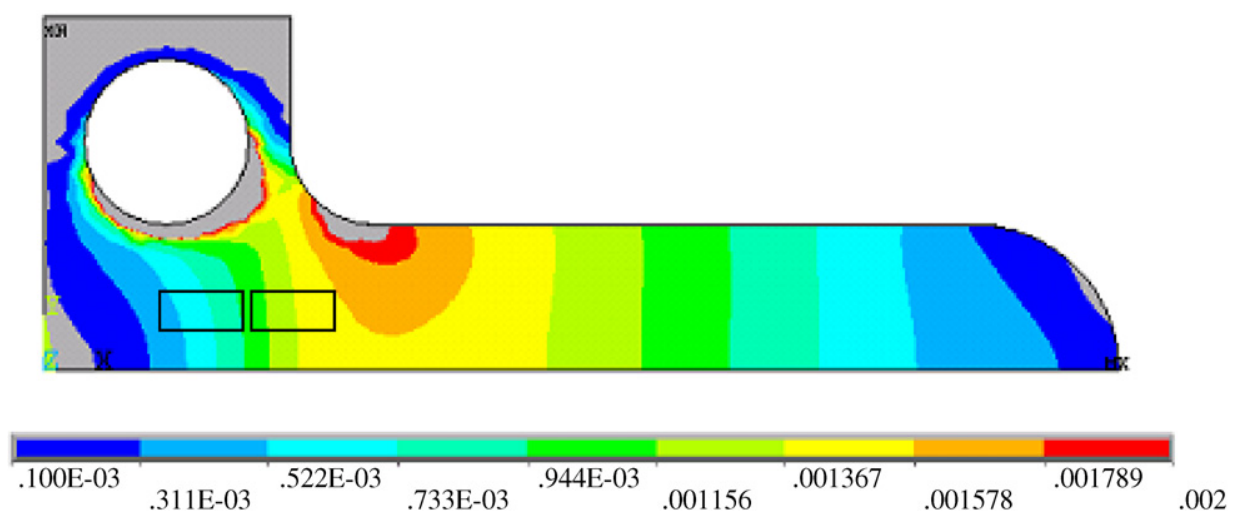

Figure 12. Strain distribution across the fingertip cantilever bolted to its support structure for $100 \mathrm{~N}$ load at beam tip. The strain range shown varies from 100 micro-strain through to 2000 micro-strain. The locations of one passive and one active thick-film resistor are highlighted.
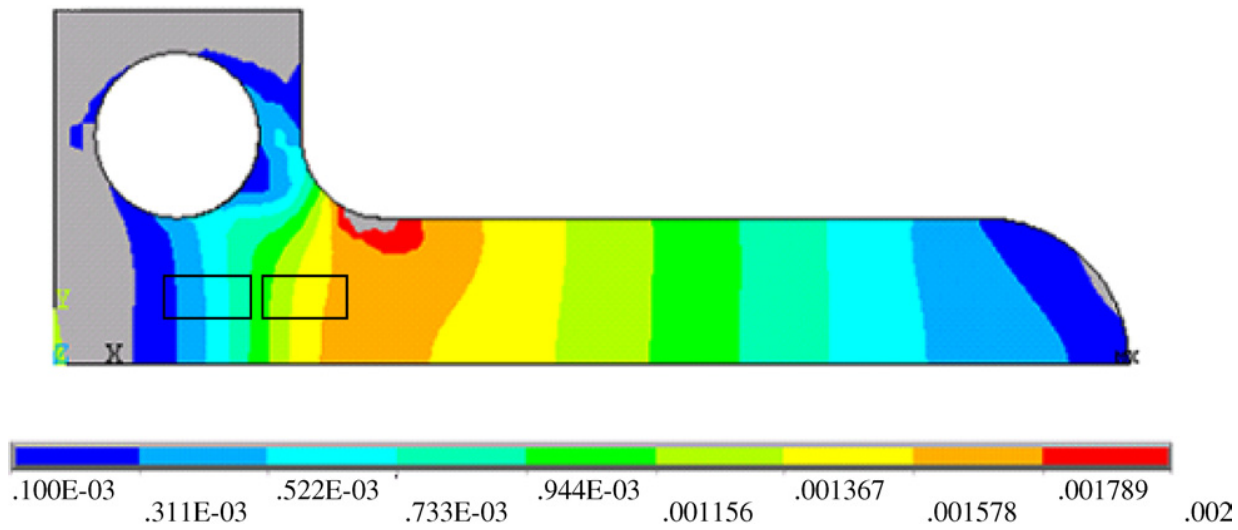

Figure 13. Strain distribution across fingertip cantilever glued to its support structure for $100 \mathrm{~N}$ load at beam tip. The strain range shown varies from 100 micro-strain through to 2000 micro-strain. The locations of one passive and one active thick-film resistor are highlighted.

temperature may be inferred from resistance in a repeatable manner and to a high degree of accuracy.

\section{Discussion}

The static force sensor shows a much lower sensitivity than that expected from theory (cf equation (4)). There are many contributory reasons for this. Firstly, the structure upon which the sensors have been fabricated is not a true cantilever beam in the mechanical sense. Ideally a cantilever beam should have a length to thickness ratio greater than 15 and possess uniform cross-sectional area along its length [27]. This is not the case in our design and so equation (2), relating the strain at the beam root to the beam tip force, is only an approximation. Secondly, the individual resistors do not have identical unstrained values for resistance and it is likely that they do not have the same gauge factor. Both of these conditions are required for the bridge measurement circuit to achieve the theoretical maximum level of measurement sensitivity.

However, the major cause for this observed difference in sensitivity can be attributed to the location of the passive resistors in the sensor design. The theory requires that these resistors experience no change in strain when the beam is deflected. An analysis of the surface strain distribution of the cantilever when the beam has been deflected reveals that the region between the cantilever mounting holes is subject to variations in strain. For example, figure 12 shows the predicted strain distribution from an ANSYS ${ }^{\mathrm{TM}}$ simulation when the beam tip is loaded with a $100 \mathrm{~N}$ force. In this model, the cantilever beam has been reduced to its lowest form of symmetry and shows that the strain experienced by the passive resistors can be as high as $50 \%$ of that experienced by the active resistors.

An ANSYS ${ }^{\mathrm{TM}}$ model was also constructed for the case where the cantilever was glued to its support structure rather than being bolted to it. Results from this simulation (shown in figure 13) reveal that whilst there is still a variation in strain in the region between the bolt holes where the passive resistors are located, the level of strain is much lower than for the case when the cantilever is bolted down to its support structure. Furthermore, the figure shows that in this instance the passive resistors of the half-bridge circuit are only subjected to approximately $35 \%$ of the strain level experienced by the active resistors. This explains why the observed force measurement sensitivity was higher when the cantilever was glued to its support compared to when it was bolted down.

\section{Conclusions}

A static (absolute) force sensor based on piezoresistive changes in thick-film resistors has been demonstrated. The force 
sensor is intended to measure and monitor forces involved in prosthetic hand grip postures, and as such has been shaped so as to mimic the average human fingertip. Force measurement sensitivities at the beam tip ranging from $6.4 \mu \mathrm{V} \mathrm{V}^{-1} \mathrm{~N}^{-1}$ up to $9.8 \mu \mathrm{V} \mathrm{V}^{-1} \mathrm{~N}^{-1}$ have been recorded, with hysteresis levels as low as $3 \%$ depending on the method of attachment of the fingertip to the remainder of the prosthetic finger, with securing by bolts being the least favourable. It has been hypothesized that if the fingertip cantilever structure were to be securely clamped to the fingertip support block then it is expected that the sensor measurement sensitivity would improve further, since the cantilever surface strain in the location of the passive resistors would remain constant as the beam were deflected. In this scenario, force measurement sensitivities should approach the theoretical value given by equation (4).

The static force sensor exhibits a positional loading error depending on the distance from the tip of the fingertip cantilever beam that the force to be resolved is acting. This equates to a $6 \%$ decrease in the measurement sensitivity for every millimetre distance from the beam tip. However, this error may be removed if contacting forces are always coupled with the tip of the cantilever beam. A simple way to ensure this criterion is to attach some form of protrusion to the tip of the beam of sufficient height such that its top surface was the proudest part of the whole fingertip structure. This protrusion would then always be the first component to be contacted as the fingers of the prosthetic hand enclose an object.

Processing problems with the piezoelectric PZT paste meant that the dynamic force sensor could not be fabricated directly on to the stainless steel fingertip structure. However, the potential for this material to act as a vibration sensor for the slip detection has been demonstrated with a substitute sensor. New devices will be constructed using other grades of stainless steel that have proven to be compatible with the PZT paste and the thick-film process to evaluate the dynamic sensor when printed directly on the cantilever fingertip [34, 35]. It is anticipated that the effective piezoelectric coefficient $d_{33}$ of these devices will be different compared to that measured here for sensors fabricated on alumina, and therefore a difference in the force detection sensitivity is also expected. This is due to potential differences in the clamping effect of these two different substrate materials [36]. The clamping effect describes how the mechanical properties of the substrate material (such as stiffness) restrict the free movement of the PZT layer in one dimension resulting in an effective lowering of the $d_{33}$ coefficient.

\section{Acknowledgment}

The authors wish to thank the Engineering and Physical Sciences Research Council (EPSRC) for their financial support under grant number GR/R95470.

\section{References}

[1] Wood C J et al 1989 Myoacoustic control of upper limb prostheses J. Assoc. Child Prosthet. Orthot. Clinics 24 2-3

[2] Zhu F and Spronck J W 1992 A capacitive tactile sensor for shear and normal force measurements Sensors Actuators A 31 115-20
[3] Chappell P H and Elliott J A 2003 Contact force sensor for artificial hands with a digital interface for a controller Meas. Sci. Technol. 14 1275-9

[4] Kyberd P J and Chappell P H 1993 A force sensor for automatic manipulation based on the Hall effect Meas. Sci. Technol. 4 281-7

[5] Heath G H 2003 Control of proportional grasping using a myokinemetric signal Technol. Disability 15 73-83

[6] Kyberd P J and Chappell P H 1992 Characterisation of an optical and acoustic touch and slip sensor for autonomous manipulation Meas. Sci. Technol. 3 969-75

[7] Tise B 1988 A compact high resolution piezoresistive digital tactile sensor Proc. IEEE Int. Conf. Rob. Auto. 7 760-4

[8] Mingrino A et al 1994 Slippage control in hand prostheses by sensing grasping forces and sliding motion IEEE/RSJ/GI Proc. Int. Conf. Intell. Robots Syst. 3 1803-9

[9] Dario P et al 1996 An integrated miniature fingertip sensor 7th Int. Symp. Micro Machine and Human Science pp 91-7

[10] Holmes P J and Loasby R G 1976 Handbook of Thick-Film Technology (Glasgow: Electrochemical Publications)

[11] Canali C et al 1980 Piezoresistive effects in thick-film resistors J. Appl. Phys. 51 3282-8

[12] Brignell J E et al 1988 Sensor applications of thick-film technology IEE Proc. I 135 77-84

[13] White N M 1989 Assessment of thick-film piezoresistors on insulated steel substrates Hybrid Circuits $2023-7$

[14] White N M and Brignell J E 1991 A planar thick-film load cell Sensors Actuators 26 313-9

[15] White N M and Turner J D 1997 Thick-film sensors: past, present and future Meas. Sci. Technol. 8 1-20

[16] Beeby S P et al 1999 Processing of PZT piezoelectric thick-films on silicon for micro electro mechanical systems J. Micromech. Microeng. 9 218-29

[17] Beeby S P et al 1999 Thick-film PZT/micromachined silicon accelerometer Electron. Lett. 35 2060-2

[18] White N M et al 2001 A novel thick-film piezoelectric micro-generator Smart Mater. Struct. 10 850-2

[19] Light C M and Chappell P H 2000 Development of a lightweight and adaptable multiple-axis hand prosthesis Med. Eng. Phys. 22 679-84

[20] Chappell P H and Kyberd P J 1991 Prehensile control of a hand prosthesis by a microcontroller J. Biomed. Eng. 13 363-9

[21] Kyberd P J and Chappell P H 1994 The Southampton Hand: An intelligent myoelectric prosthesis J. Rehabil. Res. Dev. 31 326-34

[22] Englehart K and Hudgins B 2003 A robust, real-time control scheme for multifunction myoelectric control IEEE Trans. Biomed. Eng. 50 848-54

[23] Guo G et al 1993 Optimal design of a six-bar linkage with one degree of freedom for an anthropomorphic three-jointed finger mechanism Proc. Inst. Mech. Eng. H 207 185-90

[24] Lahdenperä M 1991 Thick-film hybrid applications on stainless steel baseplate Hybrid Circuits 26 26-31

[25] Hrovat M et al 1995 A possible way to increase the gauge factors of thick film resistors J. Mater. Sci. Lett. 14 584-6

[26] Hrovat $\mathrm{M}$ et al 1995 Correlation between microstructure and gauge factors of thick film resistors J. Mater. Sci. Lett. 14 1048-51

[27] Young W C 1989 Roark's Formulas for Stress and Strains 6th edn (New York: Mc Graw-Hill)

[28] Canali C et al 1980 Strain sensitivity in thick-film resistors IEEE Trans. Compon. Hybrids Manuf. Technol. 3 421-3

[29] Prudenziati M et al 1981 Characterization of thick-film resistor strain gauges on enamel steel Sensors Actuators 2 17-27

[30] Torah R N et al 2003 A study of powder size combinations for improving piezoelectric properties of PZT thick-film devices 27th Proc. Eurosensors (Portugal) pp 360-1

[31] Torah R N et al 2004 Improving the piezoelectric properties of thick-film PZT: the influence of paste composition, powder milling process and electrode material Sensors Actuators A 110 378-84 
[32] Meitzler A H et al 1992 IEEE standard on piezoelectricity (ANSI/IEEE Std. 176-1987) Key Papers in Physics ed C Z Rosen et al (New York: American Institute of Physics) pp 227-80

[33] Dargie P et al 1998 An investigation of the effect of poling conditions on the characteristics of screen-printed piezoceramics Microelectron. Int. 15 6-10
[34] Yan T et al 2003 Thick-film PZT-metallic triple beam resonator Electron. Lett. 39 982-3

[35] Glynne-Jones P et al 2001 A method to determine the ageing rate of thick-film PZT layers Meas. Sci. Technol. 12 663-70

[36] Torah R N et al 2004 Experimental investigation into the effect of substrate clamping on the piezoelectric behaviour of thick-film PZT elements J. Phys. D: Appl. Phys. 37 1074-8 